\title{
Efectos multidimensionales emergentes de la cuarentena como consecuencia del covid-19 en los estudiantes universitarios de Bolivia
}

\author{
Karina Shirley Vera Quezada \\ Doctorante del Doctorado en Educación con Enfoque en Complejidad y \\ la Investigación Transdisciplinar (EMI), Doctorante del doctorado en \\ Ciencias Empresariales (UAB). \\ Karinaveraquezada@gmail.com \\ kveraq@post.emi.edu.bo \\ Dorian Ariel Arce Conde \\ Cursante del Doctorado en Complejidad y Educación Transdisciplinar \\ DOE-6 EMI. \\ arielarcecond@hotmail.com \\ darcec@post.emi.edu.bo \\ Bolivia
}

\section{RESUMEN}

Con la declaratoria de alerta roja en relación a la pandemia de Covid-19 para precautelar la salud de la población en el Estado plurinacional de Bolivia, comenzó a regir una cuarentena rígida para evitar que las personas salgan de sus domicilios con el objeto de evitar los contagios, Es así que, como medida inmediata, muchas instituciones educativas privadas, tomaron la iniciativa del uso de herramientas tecnológicas disponibles para garantizar la continuidad de sus actividades académicas. Súbitamente, la disposición fue alargada y muchas universidades comenzaron a emplear plataformas para garantizar la continuidad académica. El presente artículo de análisis crítico, está basado en una comprensión desde una perspectiva compleja y transdisciplinar basada en la experiencia un numero de estudiantes de universidades privadas de distintas carreras, que permitió obtener información para identificar cuáles son los efectos multidimensionales emergentes de la educación virtual que afectan el proceso de enseñanza - aprendizaje como consecuencia de la medida de confinamiento ya que tanto docentes como estudiantes, no estaban preparados para afrontar un cambio tan repentino de esta magnitud, razón por la cual los efectos se fueron suscitando paulatinamente, generando diversas consecuencias clasificadas de la siguiente manera: Efectos en el rendimiento académico por la nueva modalidad de educación a distancia, efectos emocionales y psicológicos como consecuencia de la educación virtual y efectos socioeconómicos en las familias a causa de esta modalidad en el proceso de enseñanza - aprendizaje.

Palabras clave: Emerger, complejidad, subyacer, resignificación 


\title{
Emerging multidimensional effects of quarantine as a consequence of covid-19 in university students in bolivia
}

\begin{abstract}
With the declaration of a red alert in relation to the Covid-19 pandemic to protect the health of the population in the Plurinational State of Bolivia, a rigid quarantine began to apply to prevent people from leaving their homes in order to avoid the contagions. Thus, as an immediate measure, many private educational institutions took the initiative to use available technological tools to guarantee the continuity of their academic activities. Suddenly, the provision was lengthened and many universities began to use platforms to ensure academic continuity. This critical analysis article is based on an understanding from a complex and transdisciplinary perspective based on the experience of a number of students from private universities of different careers, which allowed obtaining information to identify what are the emerging multidimensional effects of virtual education that affect the teaching learning process as a consequence of the confinement measure since both teachers and students were not prepared to face such a sudden change of this magnitude, which is why the effects were gradually aroused, generating various consequences classified as as follows: Effects on academic performance due to the new distance education modality, emotional and psychological effects as a consequence of virtual education and socioeconomic effects on families due to this modality in the teaching-learning process
\end{abstract}

Keywords: Emerge, complexity, underlie, resignification

Artículo recibido: 15 febrero 2021 Aceptado para publicación: 8 marzo 2021 Correspondencia:Karinaveraquezada@gmail.com Conflictos de Interés: Ninguna que declarar 


\section{INTRODUCCIÓN}

La cuarentena en Bolivia ocacionada por la pandemia COVID-19 es una medida establecida para evitar la propagación del virus la misma que fue declarada por el Gobierno desde el 16 de marzo de 2020 y sustentdo por el DS: 4229 de 29 de Abril de 2020 que establece que se mantine la medida de suspención temporal de clases presenciales en todos los niveles y modalidades educativas para precautelar la salud de la poblacion estudiantil, esta medida está respaldada por el Parágrafo I del Artículo 35 de la Constitución Política del Estado (CPE-2009), el cual establece que "el Estado, en todos sus niveles, protegerá el derecho a la salud, promoviendo políticas públicas orientadas a mejorar la calidad de vida, el bienestar colectivo y el acceso gratuito de la población a los servicios de salud".

Por lo citado el Ministerio de Educación determinó suspender labores educativas para evitar la propagación de este virus, siguiendo recomendaciones emitidas por la Organización Mundial de la Salud (OMS). A traves del Ministro de Eduación se anunció medidas para retornar a las actividades académicas de manera paulatina, anunciando la posibilidad de dividir cursos para las clases por turnos y de esta manera evitar la concentración de estudiantes, así también enfatizó en promover un plan de educación virtual para los profesores de manera que se pueda combinar la educación presencial con la virtual. Estas disposiciones permitieron de algún modo, dar continuidad a la actividad académica, pero no se debe dejar de lado el otro escenario, el mismo que se ve afectado como consecuencia de las convergencias complejas el cual es el sector de los estudiantes, quienes han intentado adecuarse a esta nueva metodología de formación de manera repentina, lo cual conlleva a la generación de diversos factores multidimensionales que afectan su cotidiano rendimiento académico con efectos, económicos, socio emocionales, psicológicos y otros. Como dice Basarab Nicolescu "La apertura es de tres clases: la apertura de un nivel de Realidad hacia otro nivel de Realidad, la apertura de un nivel de percepción hacia otro nivel de percepción y la apertura hacia la zona de resistencia absoluta que une el Sujeto y el Objeto. Lo desconocido, lo inesperado y lo imprevisible en un momento dado de la Historia se transforma, con el tiempo, en conocido, esperado y previsible" (Nicolescu, 1996, p. 100)

Si tomamos en cuenta este apartado desde la perspectiva del pensamiento complejo se ve explicada como el nivel de realidad inperrumpida con un suceso adverso crea niveles de realidad alterna en las 
que el individuo no esta preparado para el cambio brusco y solo a través de la adaptación transforma su nivel de realidad para adaptarse a una nueva realidad.

La ANF (Agencia de Noticias Fides) en fecha 25 de abril de 2020, publicó la iniciativa de la Organización de las Naciones Unidas para la Eduación, la ciencia y la Cultura (UNESCO) con el propósito de responder a las necesidades urgentes de la educación denominada Coalición Mundial de Educación que es una alianza de participación abierta para garantizar la continuidad de la educación para todos los estudiantes en el mundo, la cual ayudará a los países a diseñar y desplegar soluciones innovadoras y sensibles al contexto con el fin de llegar a los más desfavorecidos y a los que corren el riesgo de quedar excluidos, podemos citar para su comprencion los que expresó la Directora General de la UNESCO, Audrey Azoulay quien sostuvo que: “Actualmente existen más de mil millones de estudiantes y jóvenes de todo el mundo que están siendo afectados por el cierre de escuelas y universidades debido al brote y ahora al re brote del Covid-19 ya que de la noche a la mañana se ha ingresado en una nueva era del aprendizaje; la escolarización en el planeta, se ha convertido en algo virtual, impartida a través de las ondas y la banda ancha o simplemente se ha interrumpido"

En cada país, los gobiernos han ido implementando cada vez, diferentes modalidades de aprendizaje para la educación a distancia con la finalidad de garantizar la continuación del aprendizaje. El objetivo que busca reorientar la educación hacia el desarrollo sostenible (Agenda 2030 para el Desarrollo Sostenible), constituye la síntesis de las ambiciones de la educación, cuyo objetivo es "garantizar la educación inclusiva, equitativa y de calidad, promoviendo las oportunidades de aprendizaje durante toda la vida para todos" (UNESCO, Liderar el ODS 4 - Educación 2030, 2020.). debemos tener en cuenta que en tema educativo se debe tomar a la persona como individuo puesto que este termino contempla a la condición humana como el conjunto de situaciones ajenas o externas que lo rodean y tambien a su humana condición como lo intrínseco de cada óser haciendo de su corporeidad (unificación del ser a través de la mente-cuerpo y alma) y hacer de cada ser que sea distinto único e irrepetible tomando esta percepción podemos citar a Luengo que describe lo siguiente "Desde la educación transdisciplinaria se concibe al individuo, a los grupos sociales y a la sociedad como sistemas dinámicos complejos, que tienen la posibilidad de autoorganizarse de manera consciente". Es por ello que, todos los actores del proceso educativo, incluidos: autoridades, estudiantes, docentes, administrativos, familias e incluso la sociedad, deberán adecuarse a los nuevos cambios que exigen las condiciones como repercusión de la crisis sanitaria del Covid-19. 
Entonces partiremos de esta complejidad que dice que "Hay que enfrentar la incertidumbre puesto que vivimos una época cambiante donde los valores son ambivalentes, donde todo está ligado" (MORIN, 1997). Es por eso que, la educación del futuro debe volver sobre las incertidumbres ligadas al conocimiento. En el escenario actual, estas incertidumbres deben ser consideradas para tomar decisiones que permitan religar el pensamiento de lo que se pretendía, en función de las circunstancias que provocan efectos incalculables sobre todo en los estudiantes quienes sufren las consecuencias emergentes de la pandemia para adecuarse a los nuevos escenarios educativos en condiciones poco equitativas y con afecciones incluso psicoemocionales como resultado de ello.

Ahora bien la Dra. Gofiantini menciona: "Sin duda, las necesidades educativas hoy, para vivir en la era planetaria, demandan de todos los participantes del proceso educativo, creatividad, ingenio, capacidad de aprender y tomar decisiones para mejorar, en tanto bucle recursivo, nuestro accionar. Educar para liberar" (Gofiantini, 2016). Es entonces este el momento en el cual, será necesario resignificar la educación para darle un nuevo sentido con la participación y el involucramiento de autoridades estatales a nivel nacional e internacional, autoridades regionales, administrativos, docentes, estudiantes, padres de familia y sociedad en general para dar continuidad al proceso de formación en esta época de crisis e incertidumbre, sin dejar de lado la comprensión humana por lo que podemos decir que, "Una comprensión mutua entre humanos, tanto cercanos como extraños, es desde ahora vital para que las relaciones humanas salgan de su estado bárbaro de incompresión" (MORIN, 1997).

Por tanto, a partir de ahora, será necesario que, las universidades privadas, generen nuevas propuestas para dar continuidad a la educación, empleando una serie de iniciativas e implementando plataformas virtuales que permitan instaurar modernos e innovadores contenidos en las diferentes áreas de investigación que brinden nuevas oportunidades de desarrollo equitativas a los futuros profesionales quienes en su mayoría, están dispuestos a continuar con su formación académica para lograr su cometido. Como dice Barrero "La lógica, se ve reflejada en las normas antes mencionadas como habilidades que intentan integrar a los individuos como parte de la sociedad y como fuentes del conocimiento que permitan construir una realidad mejor, utilizando como fundamento el aprendizaje de por vida y la capacidad de integrar nuevos saberes que le permitan irse deconstruyendo" (Barreto Rozo, 2016). 
El estudio que se desgloza en el presente artículo de análisis crítico, se realiza a partir de un diagnóstico por medio de una encuesta orientada a estudiantes de universidades privadas que continuan su formación empleando diversas plataformas tecnológicas y enfrentan las incertidumbres y certezas emergentes que quedaron al descubierto en los estudiantes, docentes y sociedad en general, como consecuencia de la crisis sanitaria del COVID-19 y los abruptos cambios en el proceso de enseñanza aprendizaje al cual se ven expuestos y tuvieron que adecuarse. Además, es necesario realzar los efectos emocionales y psicológicos negativos emergentes los cuales provocan estados de tensión, estrés, temor, ansiedad, frustración, aburrimiento, afectación económica y otros.

\section{ESTRATEGIAS METODOLÓGICAS}

El presente artículo se considera de análisis crítico y pretende sistematizar experiencias mediante un estudio exploratorio por el nivel de conocimiento de la temática a abordar y luego plantear la problemática que se origina como consecuencia del virus Covid-19, asimismo, es de carácter descriptivo ya que a través de la información obtenida, se miden variables que permiten construir datos que identifican el comportamiento y la percepción de estudiantes ante las condiciones actuales y finalmente es relacional puesto que en función de los datos se puede establecer si existe o no relación con los cambios que se presentan en la conducta de quienes se ven directamente afectados por el nuevo proceso educativo que se adopta a consecuencia de la pandemia. Por último, explicativo porque se explican las causas y los efectos emergentes entre las variables analizadas, registrando las probables certezas e incertidumbres identificadas.

El método empleado fue el deductivo ya que, con la información general a partir de un cuestionario y el análisis de la situación actual, se sintetiza la misma para establecer criterios y corroborar los posibles escenarios emergentes de la actual crisis de salud que afecta al sector educativo desde diversas aristas.

Para el fin propuesto, se procedió a utilizar herramientas de recolección de datos (FORMS) como ser encuestas que contenían preguntas en tres grupos: Efectos en el rendimiento académico por la nueva modalidad de educación virtual, efectos emocionales y psicológicos como consecuencia de la educación virtual y efectos socioeconómicos en las familias a causa de la educación virtual, esta información permitió identificar los efectos multidimensionales de la educación virtual emergentes del proceso de enseñanza aprendizaje en estudiantes de instituciones educativas del ámbito privado.

A partir de ello, se realiza un análisis de las interacciones identificadas entre todos los actores que intervienen en el proceso educativo durante la pandemia del COVID-19. Como dice el coautor del 
presente artículo "la Educación no solo se transmite a través de la palabra, ya que está presente en todas nuestras emociones, sentimientos, acciones, actitudes, habilidades y destrezas esto incluye a la formación física y las prácticas culturales, como la deportiva, artística, plástica, entre otras. A ello se suma la práctica del ejemplo social como imagen global a imitarse, sobre los ideales más nobles representados por los principios y valores como ser la solidaridad y empatía entre los seres humanos" ( Arce \& Luengo, 2020) esta serie de elementos que conllevan al proceso de aprendizaje y nos leva a analizar del cambio que ocurrió dentro los niveles de la realidad ocasionadas por el suceso adverso identificada como pandemia mundial por los organismos internacionales de salud.

\section{RESULTADOS Y DISCUSIÓN}

A partir de la información obtenida mediante las herramientas de recolección de datos ya citadas, luego de su análisis se consiguió evidenciar los siguientes resultados:

En la primera pregunta menciona si los estudiantes consideran que se sentían preparados para continuar sus estudios mediante la modalidad virtual, un 33\% aseveran que No se sentían preparados, un $40 \%$ que tal vez Si y un $27 \%$ que Sí se sentían preparados. Este resultado permite aseverar que el cambio repentino al que estuvieron expuestos los estudiantes, tuvo efectos negativos y se les dificultó adaptarse a la nueva modalidad.

En cuanto a la educación virtual que reciben actualmente los estudiantes, un $58 \%$ se siente poco satisfecho con la modalidad a distancia, un $25 \%$ satisfecho, el $8 \%$ está muy satisfecho, el $7 \%$ nada satisfecho y apenas un $2 \%$ se siente totalmente satisfecho, resultado que denota un grado considerable de descontento con la enseñanza virtual.

Respecto a la pregunta de qué manera se siente afectado con la modalidad de educación virtual, los estudiantes resaltaron aspectos tales como: Frecuentes inconvenientes por la caída de la señal de internet en clases lo cual provoca constantes interrupciones, también se resalta la excesiva cantidad de tareas asignadas lo cual no les permite realizar otras actividades personales. Además, alegaron que, la modalidad virtual no permite un aprovechamiento completo en aquellas asignaturas que tienen componente práctico y laboratorio ya que las herramientas empleadas por los docentes no son suficientes para lograr la comprensión total de la materia.

Algunos estudiantes también arguyen que, en las clases virtuales, no se puede interactuar con tanta confianza como lo hacían en las clases presenciales porque no existe la conexión directa con el docente. Al mismo tiempo, la excesiva permanencia de los estudiantes en sus computadoras o 
dispositivos móviles es otro factor importante, el mismo que provoca tensión y daños en la salud óptica de los mismos. Otros consideran que las clases virtuales son más pesadas que las clases presenciales por el tiempo que deben permanecer atentos al dispositivo de comunicación ya que muchas veces el lugar donde pasan clases no es un ambiente apropiado y fácilmente logran distraerse estando constantemente expuestos a permanentes interrupciones por parte de su familia, lo cual provoca conflictos intrafamiliares y discusiones que llevan muchas veces a la depresión y ansiedad de los estudiantes.

Se evidencian también insuficiencias en la disponibilidad de dispositivos para un desarrollo académico óptimo ya que en diversas oportunidades deben compartir su equipo tecnológico con otros miembros de su familia que están en edad escolar y que también lo requieren. Este escenario denota la poca equidad en las condiciones y oportunidades de aprendizaje.

Existen constantes quejas a cerca de los métodos de enseñanza impartidos por los docentes ya que no se emplean herramientas suficientes para lograr el aprendizaje y muchos quedan con dudas, las cuales no logran ser absueltas oportunamente, sumado a esto está el nulo contacto físico con sus compañeros para colaborarse debido al aislamiento social como consecuencia de la pandemia. Estos acontecimientos también generan situaciones de depresión, angustia, temores e incertidumbre, lo cual de alguna manera puede afectar su desempeño académico y su relacionamiento interpersonal.

Se identificaron múltiples daños en la salud como consecuencia de la educación virtual que afectan la vista, así como dolores de cabeza o migrañas a causa del estrés y agotamiento físico. Asimismo, el audio en algunos casos no es el más adecuado lo que provoca un sobre esfuerzo auditivo.

En cuanto al nivel de afectación del rendimiento mediante la modalidad virtual, un $41 \%$ dicen que, Sí les afecta negativamente, un $37 \%$ tal vez se ven afectados y un $22 \%$ que no se ven afectados en su rendimiento lo cual conlleva a pensar en la dificultad que destacan al emplear esta nueva modalidad, tanto desde la perspectiva de docentes como de estudiantes debido a las condiciones inoportunas de enseñanza-aprendizaje, las mismas que, al no ser equitativas, no promueven escenarios pertinentes para un rendimiento deseable. También, consideran que su nivel de aprovechamiento no resulta óptimo mediante la modalidad virtual, respondiendo un $68 \%$ que el nivel de alteración para adecuarse a esta nueva metodología fue medio, un $22 \%$ dijo bajo y un $10 \%$ admitió que su rendimiento fue alto. Las principales causas por las cuales su rendimiento fue afectado son: el insuficiente dominio de herramientas tecnológicas por parte de los docentes en la metodología de enseñanza que emplean, 
específicamente en caso de materias prácticas y laboratorio. Además, la cantidad excesiva de tareas en las diferentes asignaturas lo cual desencadena en situaciones de estrés.

En cuanto al nivel de satisfacción con la educación virtual, un 55\% se siente poco satisfecho, solo un $20 \%$ se siente satisfecho, un $10 \%$ nada satisfecho, el $8 \%$ muy satisfecho y apenas un $7 \%$ se siente totalmente satisfecho lo cual lleva a deducir que, la mayoría de los estudiantes no consideran está modalidad como la más apropiada para el aprendizaje.

Se indagó también a cerca de las dificultades en el manejo de plataformas virtuales, a lo cual respondieron un $73 \%$ que tiene poca dificultad y un $27 \%$ no tiene ninguna dificultad. Este resultado muestra la facilidad del estudiante con la familiarización de las nuevas tecnologías lo cual es muy positivo para las circunstancias actuales a pesar de la problemática social que se atraviesa como consecuencia de la pandemia.

Finalmente se investigó a cerca de los factores socio económicos negativos como efecto de la modalidad de educación virtual y respondieron que el principal factor que afecta a una mayoría de los encuestados es la crisis económica derivada de las medidas establecidas por la cuarentena ya que se les dificulta el pago de mensualidades por diversas causas, entre ellas el desempleo, la inestabilidad laboral y económica, los costos elevados de los productos de la canasta familiar y otros. Además, otro factor a considerar es también el limitado acceso a redes WiFi lo que conlleva al uso de dispositivos móviles que genera un incremento en el presupuesto familiar por el elevado consumo de megas el mismo que afecta el bolsillo de los estudiantes y obliga a destinar un importe para inversión en servicio de internet por parte de los padres de familia. En algunos casos, no se cuenta con acceso a internet en zonas distantes lo cual provoca el abandono de un porcentaje mínimo de estudiantes y en otros casos, algunos de ellos deciden congelar el semestre o dejar de estudiar para trabajar y de esta manera, apoyar con el sustento económico de sus hogares.

A partir de ahora, es necesario que las universidades privadas, generen constantemente, nuevas propuestas para la implementación de plataformas virtuales que permitan instaurar modernos e innovadores contenidos en las diferentes áreas de investigación que brinden nuevas oportunidades de desarrollo a los futuros profesionales quienes, en su mayoría, están dispuestos a continuar con su formación académica para lograr su cometido. Además, es necesario realzar los efectos emocionales y psicológicos negativos que fluctúan provocando estados de tensión, estrés, temor, ansiedad, frustración, aburrimiento, afectación económica y otros. 
Para los futuros planes del gobierno central y las medidas a adoptar en lo que se refiere al sector educativo, será necesario considerar los objetivos para el desarrollo sostenible de la agenda 2030 que puedan hacer frente a escenarios de pobreza extrema en sectores periféricos, los cuales repercuten psicológicamente en los estudiantes y las familias donde refiere los siguiente: "Garantizar una educación inclusiva que no deje de lado a los sectores vulnerables con derecho a una educación equitativa y de calidad, asegurando el acceso en condiciones de igualdad para todos los hombres y mujeres a una formación técnica, profesional y superior de calidad". (UNESCO, Liderar el ODS 4 Educación 2030 4.3, 2020). Por esta razón, será necesario, planificar un plan de recuperación de la educación minimizando al máximo, las desigualdades emergentes como consecuencia de la crisis del aprendizaje.

Asimismo, resulta significativo considerar que, los efectos tanto a corto y mediano plazo, podrían ser devastadores. Según datos de la CEPAL, se advierte de un nuevo incremento de los índices de pobreza en Bolivia, lo cual representa un retroceso en el crecimiento económico. En la agenda 2030 para el Desarrollo Sostenible y sus 17 objetivos, se plantea la prosperidad que, a su vez, promueve la paz universal dentro de un concepto más amplio de libertad, para poner fin a la pobreza, luchar contra la desigualdad y la injusticia, estimulando durante los próximos 15 años la acción en esferas de importancia crítica para la humanidad y el planeta. "La transformación del modelo de desarrollo es urgente para una recuperación mejor con economías resilientes, inclusivas y sostenibles" (Bárcena, 2020)

El escenario pospandemia, repercute en la necesidad de considerar el cumplimiento de los objetivos de la agenda para el desarrollo sostenible 2030, específicamente, el referido a Garantizar una educación inclusiva, equitativa y de calidad y promover oportunidades de aprendizaje durante toda la vida para todos. Entonces, será necesario establecer políticas educativas que permitan fortalecer cada vez más las capacidades de producción y generar nuevas fuentes de empleo para que la crisis no desencadene en resultados catastróficos para las familias y por ende se vean afectados los estudiantes al tener que privarse de su derecho a la formación continua.

La UNESCO trabajó paralelamente con el filósofo y sociólogo francés Edgar Morin para que pueda verter ideas en relación al contexto desde la perspectiva del pensamiento complejo y a través de su libro: "Los 7 saberes necesarios para la educación del futuro" explica en su capítulo "Enseñar la condición humana" que: "La educación del futuro, deberá ser una enseñanza primera y universal centrada en la condición humana. Estamos en la era planetaria; una aventura común se apodera de 
los humanos donde quiera que estén. Estos deben reconocerse en su humanidad en común y al mismo tiempo, reconocer la diversidad cultural inherente a todo cuanto es humano". "Conocer lo humano es, principalmente situarlo en el universo y a la vez sepáralo de él.(...) Cualquier conocimiento debe contextualizar su objeto para ser pertinente. “¿Quiénes somos?” es inseparable de un “¿Dónde estamos?” ¿De dónde venimos?” y “¿A dónde vamos?”. En el entendido de considerar estos factores para entender el entramado de necesidades fluctuantes y en función de ellos, de construir y reconstruir nuevamente la educación, con el involucramiento de la sociedad en la solución de sus problemas y provocar un debate sobre las consecuencias emergentes del uso del conocimiento. Considerando que, la complejidad, desde el punto de vista de la epistemología, es una complejidad lógica que nos ayuda a concebir unidad y diversidad, orden y desorden, en el entendido que el conflicto tiende a producir algo nuevo.

Asimismo, será necesario permear los efectos disruptivos emergentes de asumir esta modalidad virtual de enseñanaza aprendizaje desde una persepectiva antagónica y complemetaria ya que son los protagonistas, tanto docentes como estudiantes y sociedad en general, quienes se ven afectados en alguna medida y solo dejando fluctuar las limitaciones a las que se ven expuestos, se impulsará hacia una nueva socio construcción del conocimiento ya que ninguno de los involucrados estaba preparado para afrontar un entramado como el que se esta viviendo.

\section{CONCLUSIÓN O CONSIDERACIONES FINALES}

En el campo de las conclusiones del estudio realizado identificados los efectos multidimensionales de la educación virtual que afectan el proceso de enseñanza aprendizaje se pudo evidenciar las siguientes consideraciones:

- Los efectos emergentes como consecuencia de la pandemia por el Covid-19, son evidentes, por un lado, se está desencadenando una crisis social paradigmática ya que permean múltiples interpretaciones en relación a los efectos de la educación a distancia los mismos que no deberían depender simplemente del contexto real, sino también deben ser consideradas las deducciones e intencionalidades de todos quienes se ven inmersos de algún modo en esta realidad como son las familias y el entorno subjetivo que constriñe a desencadenar en realidades complejas. Por otro lado, la contextualización del saber que determina la comprensión de las posibles circunstancias que rodean la probable realidad del estudiante en relación al nuevo conocimiento para una correcta valoración respecto del comportamiento o reacción hacia el uso de herramientas tecnológicas ya que 
muchos estudiantes resaltaron las deficiencias de la nueva modalidad de estudio como algo negativo y otros sí la valoraron.

- El repentino cambio en la educación tradicional por la educación virtual, ha generado en nuestro país consecuencias multivariadas a causa de la medida de cuarentena la misma que ha obligado al distanciamiento social con la finalidad de precautelar la salud y la vida de la población estudiantil. Este cambio a su vez, provoca una serie de repercusiones en la calidad educativa por la poca costumbre de emplear como medio de comunicación entre docentes y estudiantes, las herramientas tecnológicas.

- La respuesta ante los múltiples factores que condicionan la insatisfacción de los estudiantes sobre esta medida asumida como paliativo a la restricción de acudir a las aulas no se ha dejado esperar y está generando diversas consecuencias las mismas que se clasifican en tres tipos: 1. Efectos en el rendimiento académico por la nueva modalidad de educación virtual, 2. Efectos emocionales y psicológicos como consecuencia de la educación virtual y 3. Efectos socioeconómicos en las familias a causa de la educación virtual.

- En cuánto a los efectos en el rendimiento académico se puede resaltar los más trascendentales del estudio realizado que son: El cambio repentino del proceso de enseñanza aprendizaje provoca ciertas limitaciones y deficiencias en la comprensión de las diferentes asignaturas por la poca costumbre de atender a través de plataformas de comunicación tecnológica y muchos estudiantes se distraen fácilmente. Asimismo, la poca interacción entre docente-estudiante provoca algunas lagunas que no son absueltas de manera oportuna lo que ocasiona inseguridad respecto del aprendizaje, sobre todo en aquellas asignaturas que incluyen práctica y laboratorio.

- Es trascendental considerar el importante costo económico que la pandemia está imponiendo sobre la situación económica en los hogares, es así que, se identifican los efectos socio económicos ya que muchos estudiantes se ven limitados al acceso de redes WiFi y admiten pasar clases mediante su dispositivo móvil o celular haciendo uso del consumo de tarjetas y megas. En algunos casos el servicio no llega hasta sus domicilios lo cual limita una óptima comunicación. Asimismo, el servicio de internet es costoso y no todos tienen la capacidad económica de poder acceder al mismo. Y, por último, la crisis económica generada por la pandemia al ser afectado el sector de microempresarios por los cuales la inestabilidad laboral es aún mayor y esto genera inestabilidad en las familias de los estudiantes, poniendo en riesgo inclusive la continuidad de sus estudios. 
Desde una perspectiva general, pese a la multidimensionalidad de factores emergentes, la actividad académica y el rol tanto de docentes como de estudiantes empleando métodos de comunicación en entornos virtuales se está llevando adelante con la finalidad de dar continuidad al proceso formativo. Es así que la universidad como generadora de conocimiento, debe estimular el uso de estas herramientas e impulsar la innovación constante adoptando la tecnología digital, lo cual le permitirá abrir nuevos caminos complementarios a las clases presenciales además de generar espacios para que quienes desean puedan trabajar y estudiar a la vez.

Asimismo, el distanciamiento social, no puede ser un impedimento para una reconstrucción colectiva de los individuos en todo sentido, por tanto, será necesario, identificar la oportunidad y lo positivo en medio del caos y muchos sistemas se están reinventando para adecuarse a nueva normalidad de cambio en el mundo.

Finalmente, es fundamental la resignificación del rol docente quien tiene ahora el gran desafío de generar nuevos espacios de aprendizaje antagónicos y complemetarios a través de herramientas virtuales que, enfocados y aprovechados de la mejor forma posible, van a impulsar el proceso de deconstrucción, construcción y reconstrucción de la educación post y durante la emergencia sanitaria lo cual será una gran experiencia que contribuya a la transformación de una nueva sociedad generadora de conocimientos. Asimismo, se podrán proporcionar soluciones de educación alternativa, de calidad y muy flexibles, para llegar a los estudiantes que no hayan podido volver a la escuela.

Así también, las escuelas necesitarán acondicionar los espacios para poder funcionar de acuerdo con los protocolos de salud, como el distanciamiento físico, y será necesario contar con infraestructuras y suministros que permitan asegurar niveles básicos de higiene, como instalaciones de agua, saneamiento e higiene, de las que carece un alto porcentaje de las escuelas.

A su vez, durante esta crisis, la figura del docente es fundamental para enseñar a pensar, analizar, y sintetizar de manera que el estudiante pueda expresarse libremente y de esta forma, estar en posibilidades de conocer por sí mismo, aprendiendo de manera autónoma y autosuficiente. Por tanto, como dice (MORIN, 1997): "De allí la necesidad, para la educación del futuro, de una gran religazón de los conocimientos resultantes de las ciencias naturales con el fin de ubicar la condición humana en el mundo, de las resultantes de las ciencias humanas para aclarar las multidimensionalidades y complejidades humanas y la necesidad de integrar el aporte inestimable de 
las humanidades, no solamente de la filosofía y la historia, sino tambien de la literatura, la poesía, las artes, etc". "La eduación debe tener como uno de sus objetos esenciales la unión indisoluble de la condición humana entre la unidad y la diversidad de todo lo que es humano".

\section{LISTA DE REFERENCIAS}

(CPE), A. C. (7 de Febrero de 2009). Constitución Política del Estado. Texto Consitucional. El Alto Bolivia.

(s.f.). Agenda 2030 para el Desarrollo Sostenible.

Añez Chávez, J. (s.f.). DECRETO SUPREMO $N^{\circ}$ 4229. Obtenido de https://siip.produccion.gob.bo/repSIIP2/files/normativa_12345_300420205cb6.pdf

Bárcena, A. (25 de Septiembre de 2020). Secreatria Ejecutiva de la CEPAL. (C. R. ODS, Entrevistador)

Barreto Rozo, A. (2016). La interdisciplinariedad horizontal. Las formas económica, social, política y jurídica de construir realidades. Colombia: Co-Herencia.

Chavez Rodriguez, J. (2008). Apuntes para el Examen Estatal de Pedagogía. Soporte digital. La Habana.

Conde, D. A., Arce Conde, D. A., \& Luengo, A. N. (2020). Educacion Integral en la Corporeidad del Individuo. Cinergia, 5.

DE LA MORA, G. (1971). PRÓLOGO DE LA Didáctica Magna. México: Porrúa.

EDGAR, M. (1997). Los siete saberes necesarios de la educación del futuro. Multiversidad Mundo Real.

Gofiantini, V. (2016). El Kairos educativo: Re-Significar la formación docente desde la p'ractica del formador. Argentina: Laborde Editor.

Luengo, N. A., \& Martinez Alvarez, F. (2018). La educación Transdisciplinaria. Argentina: La Imprenta Ya.

Morin, E. (1992). El método IV Las ideas. Barcelona: Cátedra.

MORIN, E. (1997). Los siete saberes necesarios de la educación del futuro. Multiversidad Mundo Real.

MORIN, E. (2001). El Método I. La naturaleza de la naturaleza. (Sexta edición). Madrid, España: : CATEDRA.

Nicolescu, B. (1996). La transdisciplinariedad. Mexico: serrano.

NICOLESCU, B. (1996). La Transdisciplinariedad.Manifiesto. Mexico: Multiversidad Mundo Real. 
Robbins, S. (2009). Comportamiento Organizacional. México: Pearson Educación.

sostenible, A. 2. (s.f.). PNUD.

TORREZ, R. (2005). Obtenido de "Educación en la Sociedad de la Información"

UNESCO. (16 de Marzo de 2020). La educación en América Latina y el Caribe ante la COVID-19.

Obtenido de https://es.unesco.org/fieldoffice/santiago/covid-19-education-alc/seminarios-web

UNESCO. (Diciembre de 2020). Liderar el ODS 4 - Educación 2030. Obtenido de https://es.unesco.org/themes/liderar-ods-4-educacion-2030

UNESCO. (Diciembre de 2020). Liderar el ODS 4 - Educación 2030 4.3. Obtenido de https://es.unesco.org/themes/liderar-ods-4-educacion-2030 